\begin{tabular}{|c|l|}
\hline Title & Intramolecular Glycosylation A pproach toward Constructing the Macrocyclic Structure of Resin Glycosides \\
\hline Author(s) & Son, Sang-Hyun; Y amagiya, Natsuko; Furukawa, Jun-ichi; Sakairi, Nobuo \\
\hline Citation & $\begin{array}{l}\text { Synlett, 2009(18), 2957-2960 } \\
\text { https://doi.org/10.1055/s0029-1218272 }\end{array}$ \\
\hline Issue Date & 2009-11 \\
\hline Doc URL & http://hdl.handle.net/2115/44169 \\
\hline Rights & ○ 2009 Georg Thieme Verlag \\
\hline Type & article (author version) \\
\hline Note & LETTER \\
\hline Information & \\
\hline
\end{tabular}

Instructions for use 


\title{
Intramolecular glycosylation approach toward constructing macrocyclic structure of resin glycosides
}

\author{
Sang-Hyun Son, ${ }^{\mathrm{a}}$ Natsuko Yamagiya, ${ }^{\mathrm{a}}$ Jun-ichi Furukawa, ${ }^{\mathrm{b}}$ Nobuo Sakairi ${ }^{\mathrm{a}}$ \\ ${ }^{a}$ Graduate School of Environmental Science, Hokkaido University, Kita-ku, Sapporo \\ 060-0810, Japan \\ Fax +81(11)7062257; E-mail: nsaka@ees.hokudai.ac.jp \\ ${ }^{\mathrm{b}}$ Graduate School of Advanced Life Science, Hokkaido University, Kita-ku, Sapporo \\ 001-0021, Japan
}

\begin{abstract}
Oligosaccharide containing macrolides of resin glycosides were effectively constructed by MeOTf promoted intramolecular glycosylation of dodecyl thioglycosyl donors. Synthesis of a key disaccharide intermediate of tricolorin A and total synthesis of tricolorin $\mathrm{F}$ were successfully achieved by this approach.
\end{abstract}

Key words: resin glycoside, macrolide, tricolorin, dodecyl thioglycoside, intramolecular glycosylation

Resin glycosides are glycolipids of plant origin are one of the constituents of the Convolvulaceae (morning glory family), some of which has been used as traditional folk medicines. ${ }^{1}$ Recently, various resin glycosides with unique biological effects such as antibacterial, ${ }^{2}$ antifungal, ${ }^{3}$ cytotoxic, ${ }^{4}$ and plant growth controlling activities ${ }^{5}$ are isolated from leaves and roots of these plants. They are consisted of several deoxygenated monosaccharides and hydroxy fatty acids as an aglycon moiety. Most of the bioactive resin glycosides have unusual macrolidic structures, in which the aglycon carboxyl group is intramolecularly esterified with one of the sugar hydroxy groups. The unique structure and bioactivity attract attention of synthetic chemists. ${ }^{6}$ Synthetic studies so far reported on resin glycosides revealed that construction of the macrolide structure is a crucial step, which was conventionally carried out by intramolecular esterification between the aglycon carboxylic acid and a sugar hydroxy group under high dilution conditions. ${ }^{7}$ Recently, Fürstner developed an alternative approach employing ring closure metathesis (RCM) for this cyclization, which was applied to synthesis of various resin glycosides. ${ }^{8}$ In contrast to these two approaches, the oligosaccharide-containing macrolidic structure would be constructed by intramolecular glycosylation. In our previous studies on cycloglycan synthesis, we recognized the usefulness of thioglycosyl donors for intramolecular glycosylation. ${ }^{9}$ In this communication, we describe a model reaction of intramolecular glycosylation and application 
of this concept to synthesis of tricolorin $\mathrm{A}$ and $\mathrm{F}^{10}$ having tetra- and tri-saccharide structures, respectivly.

Applicability of intramolecular glycosylation toward a model disaccharide $\mathbf{6 R S}$ was first investigated employing our recently developed odorless dodecyl thioglycosides ${ }^{11}$ as glycosyl donors as depicted in Scheme 1. The key intermediate $\mathbf{5 R S}$ that has both glycosyl donor and acceptor functions was prepared by glycosylation and esterification of racemic methyl 11-jalapionate ${ }^{6 a} \mathbf{R} \boldsymbol{S}$ with two dodecyl thioglycosides 2 and $\mathbf{4}$. The first glycosylation was conducted with $\mathbf{1 R S}$ and the quinovoside 2 using MeOTf ${ }^{12}$ as a promoter, giving the $\square$-glycoside $\mathbf{3 R S}$ in $85 \%$ yield. After saponification of $\mathbf{3 R S}$, the resulting acid was esterified with 4 to give $\mathbf{5 R S}$ in $69 \%$ yield. ${ }^{14}$ The precursor $\mathbf{5 R S}$ was subjected to intramolecular glycosylation under the conditions summarized in Table 1. Comparison with two promoters, NIS̃TfOH ${ }^{13}$ and MeOTf (entries 2 and 5), showed that the former reaction gave $\mathbf{6 R \boldsymbol { S } ^ { 1 4 }}$ in slightly higher yield with an almost same ratio of the diastereomer. Although increasing the substrate concentration resulted in rapid consumption of $\mathbf{5 R S}$ and production of various polar products, almost same yield (2735\%) of the desired S-isomer $\mathbf{6 S}$ was obtained. These results suggest that the $R$-isomer of $\mathbf{5 R S}$ easily undergoes intermolecular glycosylation to afford polymeric compounds. The best results were obtained at the substrate concentration of 2.2 $\mathrm{mM}$ with $10 \mathrm{~mol}$ equiv promoter, giving $\mathbf{6 R S}$ in $69 \%$ yield with a diastereomer ratio of $R / S=$ 52 : 48. For structural elucidation of the products, the corresponding $S$-isomer $6 S$ was synthesized from $1 S$.

Encouraged by these successful results of the model experiments, we next examined the synthesis of a disaccharide constituent of tricolorin A. As shown in Scheme 2, the D-fucosyl donor 7 was first coupled with enantiomerically pure aglycon ${ }^{7 a} 1 S$ using MeOTf as a promoter giving 8 in $81 \%$, which was subsequently saponified and condensed with the glucosyl donor 11. Although we chose trichloroethoxycarbonyl (Troc) group as a temporary protective group of $\mathbf{1 1}$, its migration during the condensation reaction resulted in low isolated yield of $12 .{ }^{14}$ The key intermediate 12 thus obtained was subjected to similar MeOTf promoted intramolecular glycosylation in $\mathrm{CH}_{2} \mathrm{Cl}_{2}$ at a substrate concentration of $2.1 \mathrm{mM}$, giving the macrolide ${ }^{14} 13$ in $67 \%$ isolated yield. The coupling constant $(7.6 \mathrm{~Hz}$ ) between $\mathrm{H}-1^{\prime}$ and $2^{\prime}$ suggested that the anomeric configuration of newly formed glycosidic bond was $\beta$.

Our attention next focused on the synthesis of a trisaccharidic resin glycoside, tricolorin $\mathrm{F}^{10}$ having a 21 membered ring. The D-fucosyl acceptor 9, which was prepared by Zemplén de-O-benzoylation of $\mathbf{8}$, underwent MeOTf promoted glycosylation with the donor $\mathbf{1 4}$ to give the disaccharide $\mathbf{1 5}$ in $83 \%$ yield. In order to transform into the substrate $\mathbf{1 9}$ for 
intramolecular glycosylation, the ester functions in $\mathbf{1 5}$ were hydrolyzed with aq. $\mathrm{NaOH}$. Subsequently, the resulting carboxylic acid $\mathbf{1 6}$ was to be coupled with the thioquinovoside $\mathbf{1 7}$. Stoichiometric reaction between $\mathbf{1 6}$ and $\mathbf{1 7}$ gave a complex mixture containing the desired $\mathbf{1 9}$ only in 9\% yield probably due to polymerization. This problem was overcome by use of excess alcohol 17 (5 mol equiv) to give 18 in $72 \%$ yield. In its ${ }^{1} \mathrm{H}$ NMR spectrum, a signal assignable to $\mathrm{H}-2$ of the quinovose residue was observed at lower magnetic field of $\delta 4.95$ as a triplet $(J=9.6 \mathrm{~Hz})$, suggesting that the hydroxy group at this position was esterified. The next intramolecular glycosylation of $\mathbf{1 8}$ using similar MeOTf method found to proceed smoothly in $\mathrm{CH}_{2} \mathrm{Cl}_{2}$ at the substrate concentration of $1.8 \mathrm{mM}$, giving known fully protected derivative $19^{7 \mathrm{a}}$ of tricolorin $\mathrm{F}$ in $70 \%$ yield. Its structure was confirmed mainly by NMR spectroscopy; ${ }^{14} \mathrm{H}-1{ }^{\prime \prime}$ and $\mathrm{H}-2{ }^{\prime \prime}$ were observed at doublet $(J=10.8 \mathrm{~Hz})$ and $\delta 5.01$, respectively, showed that newly formed $\beta$-D-qunovosyl linkage and the 1", 2 -macrolide structure. It was noteworthy that $\mathrm{H}-1$ and $\mathrm{H}-1^{\prime}$ observed at $\delta 5.06$ and 4.42 as broad doublets probably due to the constrained structure of its trisaccharide containing macrolide structure. Finally global deprotection of $\mathbf{1 9}$ was successfully attained by a two-step procedure involving hydrolysis of the acetal group with $3 \% \mathrm{HCl} /$ methanol and catalytic hydrogenolysis of the O-benzyl groups over $\mathrm{Pd} / \mathrm{C}$ in $\mathrm{MeOH}$, giving tricolorin $\mathrm{F} \mathbf{2 0}^{\mathrm{7a}}$ in almost quantitative yield.

In conclusion we have succeeded in synthesis of the macrolidic structures of resin glycosides through novel intramolecular glycosylation approach. Combination with macrolactonization and RCM, the new methodology would provide various synthetic analogues useful for a systematic survey of the biological functions of resin glycosides.

\section{Acknowledgments}

We would like to thank Ms A. Maeda and Ms H. Matsumoto at Center for Instrumental Analysis, Hokkaido University for measuring elemental analyses, and Ms S. Oka at the same center for recoding MS spectra.

\section{References and Notes}

(1) Pereda-Miranda, R.; Bah, M. Curr. Top. Med. Chem. 2003, 3, 111-131.

(2) Cherigo, L.; Pereda-Miranda, R.; Fragoso-Serrano, M.; Jacobo-Herrera, N.; Kaatz, G. W.; Gibbons, S. J. Nat. Prod. 2008, 71, 1037-1045.

(3) Barnes, C. C.; Smalley, M. K.; Manfredi, K. P.; Kindscher, K.; Loring, H.; Sheeley, D. M. J. Nat. Prod. 2003, 66, 1457-1462.

(4) Pereda-Miranda, R.; Mata, R.; Anaya, A. L.; Wickramaratne, D. B. M.; Pezzuto, J. M.; Kinghorn, A. D. J. Nat. Prod. 1993, 56, 571-582. 
(5) Leon, I.; Enriquez, R. G.; Gnecco, D.; Villarreal, M. L.; Cortes, D. A.; Reynolds, W. F.; Yu, M. J. Nat. Prod. 2004, 67, 1552-1556.

(6) (a) Fürstner, A. Euro. J. Org. Chem. 2004, 943-958. (b) Furukawa, J.; Sakairi, N. Trends Glycosci. Glycotechnol. 2001, 13, 110.

(7) (a) Brito-Arias, M.; Pereda-Miranda, R.; Heathcock, C. H. J. Org. Chem. 2004, 69, 4567-4570. (b) Zhu, X.-M.; He, L.-L.; Yang, G.-L.; Lei, M.; Chen, S.-S.; Yang, J.-S. Synlett. 2006, 3510-3512 and references cited therein.

(8) (a) Fürstner, A.; Müller, T. J. Org. Chem. 1998, 63, 424்425. (b) Fürstner, A.; Müller, T. J. Am. Chem. Soc. 1999, 121, 78147821. (c) Fürstner, A.; Jea njean, F.; Razon, P. Angew. Chem. Int. Ed. 2002, 41, 20972101. (d) Fürstner, A.; Jeanjean, F.; Razon, P.; Wirtz, C.; Mynott, R. Chem. Eur. J. 2003, 9, 307319 (e) Fürstner, A.; Jeanjean, F.; Razon, P.; Wirtz, C.; Mynott, R. Chem. Eur. J. 2003, 9, 3200326.

(9) (a) Sakairi, N.; Wang, L.-X.; Kuzuhara, H. J. Chem. Soc., Chem. Commun. 1991, 2892290.

(b) Sakairi N.; Kuzuhara, H. J. Chem. Soc., Chem. Commun. 1993, 18741875.

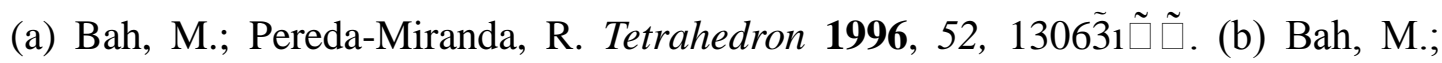

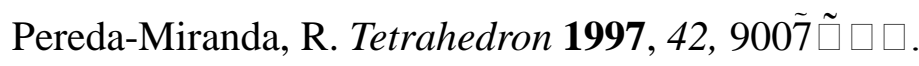

(11) ～(a) Hasegawa, J.; Hamada, M.; Miyamoto, T.; Nishide, K.; Kajimoto, T.; Uenishi, J.; Node, M. Carbohydr. Res. 2005, 340, 23602368. (b) Matsui, H.; Furukawa, J.; Awano, T.; Nishi, N.; Sakairi, N. Chem. Lett. 2000, 326327. (c) Son, S.-H.; Tano, C.; Furukawa, J.; Furuike, T.; Sakairi, N. Org. Biomol. Chem. 2008, 6, 144111449. (d) Son, S.-H.; Tano, C.; Furuike, T.; Sakairi, N. Tetrahedron Lett. 2008, 49, 52895292. (e) Son, S.-H.; Tano, C.; Furuike, T.; Sakairi, N. Carbohydr. Res. 2009, 344, $28 \tilde{5} 290$.

(12) Lönn, H. Carbohydr. Res. 1985, 139, $11 \tilde{5} 121$.

(13) Mootoo, D. R.; Konradsson, P.; Udodong, U.; Fraser-Reid, B. J. Am. Chem. Soc. 1988, $110,558 \tilde{3} 5584$.

(14) All new compounds had satisfactory data for elemental analysis or high resolution mass spectrum. Selected physical data.

Compound 5RS: ${ }^{1} \mathrm{H}$ NMR (300 MHz, $\left.\mathrm{CDCl}_{3}\right): \delta=8.027 .25\left(\mathrm{~m}, 20 \mathrm{H}, \mathrm{CH}_{\text {arom }}\right.$ ), 5.45 (t, 1 $\left.\mathrm{H}, J_{3^{\prime}, 4^{\prime}}=9.4 \mathrm{~Hz}, \mathrm{H}-3^{\prime}\right), 5.19$ (t, $\left.1 \mathrm{H}, J_{2^{\prime}, 3^{\prime}}=9.8 \mathrm{~Hz}, \mathrm{H}-2^{\prime}\right), 4.974 .61\left(\mathrm{~m}, 6 \mathrm{H}, \mathrm{PhCH}_{2}\right)$, 4.57 (d, $1 \mathrm{H}, J_{1^{\prime}, 2^{\prime}}=10.0 \mathrm{~Hz}, \mathrm{H}-1^{\prime}$ ), 4.25 (d, $\left.1 \mathrm{H}, J_{1,2}=7.1 \mathrm{~Hz}, \mathrm{H}-1\right), 3.613 .48$ (m, $4 \mathrm{H}$, H-2, 3, 5', OCH), 3.4133.33 (m, 2 H, H-5, 4'), 3.20 (t, 1 H, J 4, $=8.6$ Hz, H-4), 2.692 .63 (m, $2 \mathrm{H}, \mathrm{SCH}_{2}$ ), 2.26 (s, $1 \mathrm{H}, \mathrm{OH}$ ), 2.09 (t, $2 \mathrm{H}, J=7.3 \mathrm{~Hz}, \mathrm{CH}_{2} \mathrm{COO}$ ), $1.57 \square \square(\mathrm{m}, 56 \mathrm{H}$, $\mathrm{H}-6,6^{\prime}, 22 \times \mathrm{CH}_{2}, 2 \times \mathrm{CH}_{3}$ ). Although $\mathbf{5 R}$ and $\mathbf{5 S}$ were not distinguished by both ${ }^{1} \mathrm{H}$ and ${ }^{13} \mathrm{C}$ NMR spectra, the diastereomer ratio of almost $1: 1$ was deduced by careful HPTLC (Merck) analysis on silica gel using hexanẽEtOAc $(2: 1)$. 
Compound 6R: $[\alpha]_{\mathrm{D}}+13.3\left(\right.$ c 1.05, $\left.\mathrm{CHCl}_{3}\right) .{ }^{1} \mathrm{H}$ NMR (300 MHz, $\left.\mathrm{CDCl}_{3}\right): \delta=7.907 .19(\mathrm{~m}$, $20 \mathrm{H}, \mathrm{CH}_{\text {arom }}$ ), 5.41 (t, $1 \mathrm{H}, J_{3^{\prime}, 4^{\prime}}=8.6 \mathrm{~Hz}, \mathrm{H}-3^{\prime}$ ), 5.23 (t, $1 \mathrm{H}, J_{2^{\prime}, 3^{\prime}}=7.7 \mathrm{~Hz}, \mathrm{H}-2^{\prime}$ ), 5.14 (d, $\left.1 \mathrm{H}, J_{1^{\prime}, 2^{\prime}}=7.7 \mathrm{~Hz}, \mathrm{H}-1^{\prime}\right), 4.754 .48\left(\mathrm{~m}, 6 \mathrm{H}, \mathrm{PhCH}_{2}\right.$ ), 4.39 (d, $1 \mathrm{H}, J_{1,2}=7.7 \mathrm{~Hz}, \mathrm{H}-1$ ),

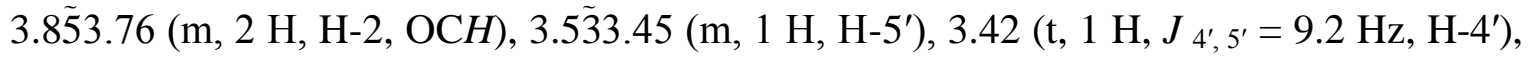
3.41 (t, $\left.1 \mathrm{H}, J_{3,4}=8.5 \mathrm{~Hz}, \mathrm{H}-3\right), 3.2903 .22$ (m, $\left.1 \mathrm{H}, \mathrm{H}-5\right), 3.11$ (t, $\left.1 \mathrm{H}, J_{4,5}=9.1 \mathrm{~Hz}, \mathrm{H}-4\right)$, 2.29̃2.08 (m, $\left.2 \mathrm{H}, \mathrm{CH}_{2} \mathrm{COO}\right), 1.6711 .21\left(\mathrm{~m}, 30 \mathrm{H}, \mathrm{H}-6,6^{\prime}, 12 \times \mathrm{CH}_{2}\right), 0.88$ (t, $3 \mathrm{H}, J=6.7$ $\left.\mathrm{Hz}, \mathrm{CH}_{2} \mathrm{CH}_{3}\right) .{ }^{13} \mathrm{C}$ NMR $\left(75.5 \mathrm{MHz}, \mathrm{CDCl}_{3}\right): \delta=172.4,165.4,138.5,138.1,133.4,130.2$, 130.1, 129.0, 128.8, 128.4, 128.4, 128.2, 128.0, 99.6, 95.6, 86.4, 84.4, 82.9, 81.0, 76.2, 75.8, 75.6, 75.4, 74.9, 71.2, 71.1, 34.9, 34.5, 34.5, 32.6, 31.2, 29.4, 28.3, 28.0, 27.9, 26.9, 23.1, 18.5, 18.4, 14.6. HRMS (FAB): $\mathrm{m} / \mathrm{z}$ calcd for $\mathrm{C}_{56} \mathrm{H}_{72} \mathrm{O}_{11}[\mathrm{M}+\mathrm{Na}]^{+}$: 943.4995; found: 943.4995 .

Compound 6S: $[\alpha]_{\mathrm{D}}+25.6\left(\right.$ c 0.20, $\left.\mathrm{CHCl}_{3}\right) .{ }^{1} \mathrm{H}$ NMR (300 MHz, $\left.\mathrm{CDCl}_{3}\right): \delta=7.927 .17(\mathrm{~m}$, $20 \mathrm{H}, \mathrm{CH}_{\text {arom }}$ ), 5.42 (t, $1 \mathrm{H}, J_{3^{\prime}, 4^{\prime}}=8.6 \mathrm{~Hz}, \mathrm{H}-3^{\prime}$ ), 5.23 (t, $1 \mathrm{H}, J_{2^{\prime}, 3^{\prime}}=8.3 \mathrm{~Hz}, \mathrm{H}-2^{\prime}$ ), 5.07 (d, $\left.1 \mathrm{H}, J_{1^{\prime}, 2^{\prime}}=7.6 \mathrm{~Hz}, \mathrm{H}-1^{\prime}\right), 4.754 .46\left(\mathrm{~m}, 6 \mathrm{H}, \mathrm{PhCH}_{2}\right.$ ), 4.24 (d, $1 \mathrm{H}, J_{1,2}=7.8 \mathrm{~Hz}, \mathrm{H}-1$ ), 3.82 (dd, $1 \mathrm{H}, J=8.0 \mathrm{~Hz}, J_{2,3}=9.3 \mathrm{~Hz}, \mathrm{H}-2$ ), 3.533 .46 (m, $2 \mathrm{H}, \mathrm{H}-5^{\prime}, \mathrm{OCH}$ ), 3.40 (t, $1 \mathrm{H}$, $\left.J_{4^{\prime}, 5^{\prime}}=9.2 \mathrm{~Hz}, \mathrm{H}-4^{\prime}\right), 3.40$ (t, $1 \mathrm{H}, J_{3,4}=8.4 \mathrm{~Hz}, \mathrm{H}-3$ ), $3.3 \tilde{1} 3.26$ (m, $\left.1 \mathrm{H}, \mathrm{H}-5\right), 3.11$ (t, 1 $\left.\mathrm{H}, J_{4,5}=9.2 \mathrm{~Hz}, \mathrm{H}-4\right), 2.19$ (s, $\left.2 \mathrm{H}, \mathrm{CH}_{2} \mathrm{COO}\right), 1.591 .22\left(\mathrm{~m}, 30 \mathrm{H}, \mathrm{H}-6,6\right.$ 6', $12 \times \mathrm{CH}_{2}$ ), $0.89\left(\mathrm{t}, 3 \mathrm{H}, J_{1,2}=6.4 \mathrm{~Hz}, \mathrm{CH}_{2} \mathrm{CH}_{3}\right) .{ }^{13} \mathrm{C} \mathrm{NMR}\left(75.5 \mathrm{MHz}, \mathrm{CDCl}_{3}\right): \delta=172.4,165.5$, 138.5, 138.2, 133.4, 130.2, 130.1, 129.0, 128.9, 128.8, 128.4, 128.3, 128.3, 128.2, 128.0, 102.3, 99.5, 86.1, 84.1, 83.2, 82.2, 76.5, 75.8, 75.6, 75.5, 75.1, 71.5, 70.9, 35.3, 35.0, 34.6, 32.5, 31.6, 29.8, 28.4, 28.1, 27.3, 25.7, 25.4, 23.4, 23.1, 18.4, 14.6. HRMS (FAB): $m / z$ calcd for $\mathrm{C}_{56} \mathrm{H}_{72} \mathrm{O}_{11}[\mathrm{M}+\mathrm{Na}]^{+}$: 943.4995; found: 943.4995 .

Compound 12: $[\alpha]_{\mathrm{D}} 7.2\left(c \mathrm{c} 0.76, \mathrm{CHCl}_{3}\right) .{ }^{1} \mathrm{H}$ NMR (300 MHz, acetone- $\left.d_{6}\right): \delta=7.407 .25$ (m, $10 \mathrm{H}, 2 \mathrm{CH}_{\text {arom}}$ ), 5.40 (t, $\left.1 \mathrm{H}, J_{3^{\prime}, 4^{\prime}}=8.9 \mathrm{~Hz}, \mathrm{H}-3^{\prime}\right), 4.974 .57\left(\mathrm{~m}, 6 \mathrm{H}, \mathrm{PhCH}_{2}\right.$, $\mathrm{Cl}_{3} \mathrm{CCH}_{2}$ ), 4.82 (d, $\left.1 \mathrm{H}, J_{1^{\prime}, 2^{\prime}}=10.0 \mathrm{~Hz}, \mathrm{H}-1^{\prime}\right), 4.73$ (t, $\left.1 \mathrm{H}, J_{2^{\prime}, 3^{\prime}}=9.6 \mathrm{~Hz}, \mathrm{H}-2^{\prime}\right), 4.22$ (d, $\left.1 \mathrm{H}, J_{1,2}=8.1 \mathrm{~Hz}, \mathrm{H}-1\right), 4.03\left(\mathrm{dd}, 1 \mathrm{H}, J=2.2 \mathrm{~Hz}, J_{4,5}=6.0 \mathrm{~Hz}, \mathrm{H}-4\right), 4.0003 .96(\mathrm{~m}, 1 \mathrm{H}$, H-3), 3.91 (dd, $1 \mathrm{H}, J=2.1 \mathrm{~Hz}, J=6.5 \mathrm{~Hz}, \mathrm{H}-5$ ), 3.863 .75 (m, $4 \mathrm{H}, \mathrm{H}-4$ ', 5', 6'a, 6'b), $3.6 \tilde{3} 3.60(\mathrm{~m}, 1 \mathrm{H}, \mathrm{CHO}), 3.35\left(\mathrm{t}, 1 \mathrm{H}, J_{2,3}=7.6 \mathrm{~Hz}, \mathrm{H}-2\right), 2.852 .65\left(\mathrm{~m}, 2 \mathrm{H}, \mathrm{S} \mathrm{CH}_{2}\right)$, 2.3َ̃2.20 (m, $\left.2 \mathrm{H}, \mathrm{CH}_{2} \mathrm{COO}\right), 1.5 \tilde{2} 1.28\left(\mathrm{~m}, 53 \mathrm{H}, \mathrm{H}-6, \mathrm{C}\left(\mathrm{CH}_{3}\right)_{2}, 22 \times \mathrm{CH}_{2}\right), 0.9 \tilde{1} 0.86(\mathrm{~m}, 6$ $\mathrm{H}, 2 \times \mathrm{CH}_{3}$ ). Column chromatography of the mixture gave crude 12 contaminated with small amount of an unknown product in ca. 60\% yield and a mixture of $\mathbf{1 1}$ its 3-O-Troc derivative. Repeated column chromatography gave pure 12 in $12 \%$ yield.

Compound 13: $[\alpha]_{\mathrm{D}}+2.1\left(c\right.$ 0.33, $\left.\mathrm{CHCl}_{3}\right) .{ }^{1} \mathrm{H}$ NMR (300 MHz, acetone- $\left.d_{6}\right): \delta=7.447 .29$ (m, $10 \mathrm{H}, \mathrm{CH}_{\text {arom }}$ ), 5.48 (t, $\left.1 \mathrm{H}, J_{3^{\prime}, 4^{\prime}}=8.6 \mathrm{~Hz}, \mathrm{H}-3^{\prime}\right), 5.36\left(\mathrm{~d}, 1 \mathrm{H}, J_{1^{\prime}, 2^{\prime}}=7.6 \mathrm{~Hz}, \mathrm{H}-1^{\prime}\right)$, $5.084 .63\left(\mathrm{~m}, 6 \mathrm{H}, \mathrm{PhCH}_{2}, \mathrm{Cl}_{3} \mathrm{CCH}_{2}\right.$ ), 4.57 (dd, $\left.1 \mathrm{H}, J=7.6 \mathrm{~Hz}, J_{2^{\prime}, 3^{\prime}}=8.8 \mathrm{~Hz}, \mathrm{H}-2^{\prime}\right), 4.23$ (d, $1 \mathrm{H}, J_{1,2}=8.4 \mathrm{~Hz}, \mathrm{H}-1$ ), 4.11 (dd, $1 \mathrm{H}, J=5.3 \mathrm{~Hz}, J_{4,5}=7.0 \mathrm{~Hz}, \mathrm{H}-4$ ), 4.05 (dd, $1 \mathrm{H}$, $\left.J=2.0 \mathrm{~Hz}, J_{3,4}=5.3 \mathrm{~Hz}, \mathrm{H}-3\right), 3.9 \tilde{5} 3.84\left(\mathrm{~m}, 5 \mathrm{H}, \mathrm{H}-2,5,4^{\prime}, 6^{\prime} \mathrm{a}, 6^{\prime} \mathrm{b}\right), 3.7 \tilde{1} 3.65$ (m, $1 \mathrm{H}$, H-5'), 3.553.49 (m, 1 H, CHO), 2.332.00 (m, $\left.2 \mathrm{H}, \mathrm{CH}_{2} \mathrm{COO}\right), 1.531 .24$ (m, $33 \mathrm{H}, \mathrm{H}-6$, 
$\left.\mathrm{C}\left(\mathrm{CH}_{3}\right)_{2}, 12 \times \mathrm{CH}_{2}\right), 0.86\left(\mathrm{t}, 3 \mathrm{H}, \mathrm{J}=6.8 \mathrm{~Hz}, \mathrm{CH}_{2} \mathrm{CH}_{3}\right) .{ }^{13} \mathrm{C} \mathrm{NMR}(75.5 \mathrm{MHz}$, acetone- $\left.d_{6}\right): \delta=171.8,153.4,139.1,138.8,128.6,128.3,128.2,128.0,127.7,109.6$, 101.5, 97.0, 95.3, 81.2, 80.5, 80.2, 77.0, 76.9, 75.5, 75.1, 74.7, 74.5, 73.5, 68.6, 68.3, 35.4, 35.2, 34.5, 32.1, 31.1, 30.1, 29.9, 29.6, 29.3, 29.1, 28.8, 28.6, 28.3, 28.2, 27.9, 27.4, 26.3, 25.4, 25.1, 24.2, 22.8, 16.7, 13.9. HRMS (FAB): $\mathrm{m} / \mathrm{z}$ calcd for $\mathrm{C}_{48} \mathrm{H}_{67} \mathrm{Cl}_{3} \mathrm{O}_{13}[\mathrm{M}+\mathrm{Na}]^{+}$: 979.3545; found: 979.3542 .

Compound 19: $[\alpha]_{\mathrm{D}} 20.5\left(c\right.$ 0.064, $\left.\mathrm{CHCl}_{3}\right) .{ }^{1} \mathrm{H}$ NMR $\left(600 \mathrm{MHz}\right.$, acetone- $\left.d_{6}\right): \delta=7.4 \tilde{1} 7.20$ (m, $25 \mathrm{H}, \mathrm{CH}_{\text {arom }}$ ), 5.06 (d, $1 \mathrm{H}, J_{1^{\prime}, 2^{\prime}}=7.4 \mathrm{~Hz}, \mathrm{H}-1^{\prime}$ ), 5.01 (s, $\left.1 \mathrm{H}, \mathrm{H}-1^{\prime \prime}\right), 5.01$ (t, $1 \mathrm{H}, J_{2^{\prime \prime}}$, $\left.3^{\prime \prime}=4.5 \mathrm{~Hz}, \mathrm{H}-2^{\prime \prime}\right), 5.014 .61\left(\mathrm{~m}, 10 \mathrm{H}, \mathrm{PhCH}_{2}\right), 4.45$ (t, $\left.1 \mathrm{H}, J_{3,4}=6.3 \mathrm{~Hz}, \mathrm{H}-3\right), 4.42$ (d, $\left.1 \mathrm{H}, J_{1,2}=6.4 \mathrm{~Hz}, \mathrm{H}-1\right), 4.12\left(\mathrm{dd}, 1 \mathrm{H}, J=2.0 \mathrm{~Hz}, J_{4,5}=5.7 \mathrm{~Hz}, \mathrm{H}-4\right), 3.983 .91(\mathrm{~m}, 1 \mathrm{H}$, H-5), 3.94 (t, 1 H, $\left.J_{2,3}=6.4 \mathrm{~Hz}, \mathrm{H}-2\right), 3.803 .74$ (m, $\left.2 \mathrm{H}, \mathrm{H}-6^{\prime} \mathrm{a}, 6^{\prime} \mathrm{b}\right), 3.69$ (t, $1 \mathrm{H}, J_{3^{\prime}, 4^{\prime}}=$ $\left.7.7 \mathrm{~Hz}, \mathrm{H}-3^{\prime}\right), 3.64$ (m, $\left.1 \mathrm{H}, \mathrm{H}-3^{\prime \prime}\right), 3.61$ (t, $\left.1 \mathrm{H}, J_{2^{\prime}, 3^{\prime}}=7.4 \mathrm{~Hz}, \mathrm{H}-2^{\prime}\right), 3.58$ (t, $1 \mathrm{H}, J_{4^{\prime}, 5^{\prime}}=$ $7.3 \mathrm{~Hz}, \mathrm{H}-4^{\prime}$ ), 3.56 (m, $\left.2 \mathrm{H}, \mathrm{H}-5^{\prime \prime}, \mathrm{CHO}\right), 3.4 \tilde{6} 3.42$ (m, $\left.1 \mathrm{H}, \mathrm{H}-5^{\prime}\right), 3.32$ (t, $1 \mathrm{H}, \mathrm{J}_{4^{\prime \prime}}, 5^{\prime \prime}=$ $\left.9.1 \mathrm{~Hz}, \mathrm{H}-4^{\prime \prime}\right), 2.13$ (t, $2 \mathrm{H}, J=7.6 \mathrm{~Hz}, \mathrm{CH}_{2} \mathrm{COO}$ ), 1.7000 .85 (m, $39 \mathrm{H}, \mathrm{H}-6,6^{\prime \prime}, 12 \times \mathrm{CH}_{2}$, $\left.\mathrm{C}\left(\mathrm{CH}_{3}\right)_{2}, \mathrm{CH}_{2} \mathrm{CH}_{3}\right) .{ }^{13} \mathrm{C}$ NMR $\left(75.5 \mathrm{MHz}\right.$, acetone- $\left.d_{6}\right): \delta=172.6,138.9,138.7,138.5$, 138.3, 129.0, 128.9, 128.8, 128.6, 128.5, 128.3, 128.2, 128.1, 127.9, 127.6, 110.2, 102.9, 100.8, 83.8, 78.7, 77.7, 75.9, 75.8, 75.6, 75.5, 75.4, 74.0, 72.0, 68.8, 36.6, 35.4, 34.5, 32.5, 30.2, 30.1, 29.4, 28.2, 28.1, 28.0, 26.9, 25.5, 23.1, 18.7, 17.3, 14.6. HRMS (FAB): $m / z$ calcd for $\mathrm{C}_{72} \mathrm{H}_{96} \mathrm{O}_{15}[\mathrm{M}+\mathrm{H}]^{+}:$1199.6672; found: 1199.6640 . 


\section{Scheme 1}

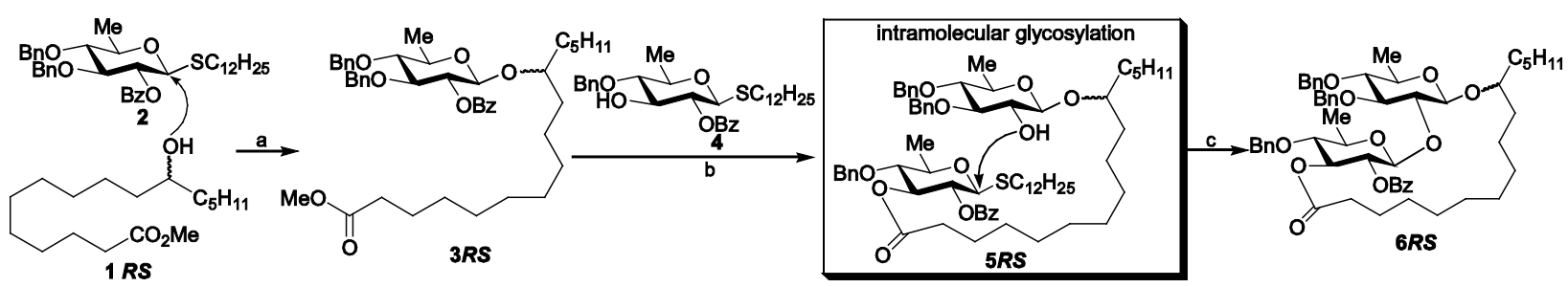

Scheme 1 Intramolecular glycosylation toward a model disaccharide 6RS. Reagents and conditions: (a) MeOTf, MS $4 \AA$ A $\mathrm{CH}_{2} \mathrm{Cl}_{2}$, r.t., 1.5 h, 85\%; (b) $\mathrm{NaOH}$, THF-EtOH, $50{ }^{\circ} \mathrm{C}, 7$ h; then 4,

\section{Scheme 2}

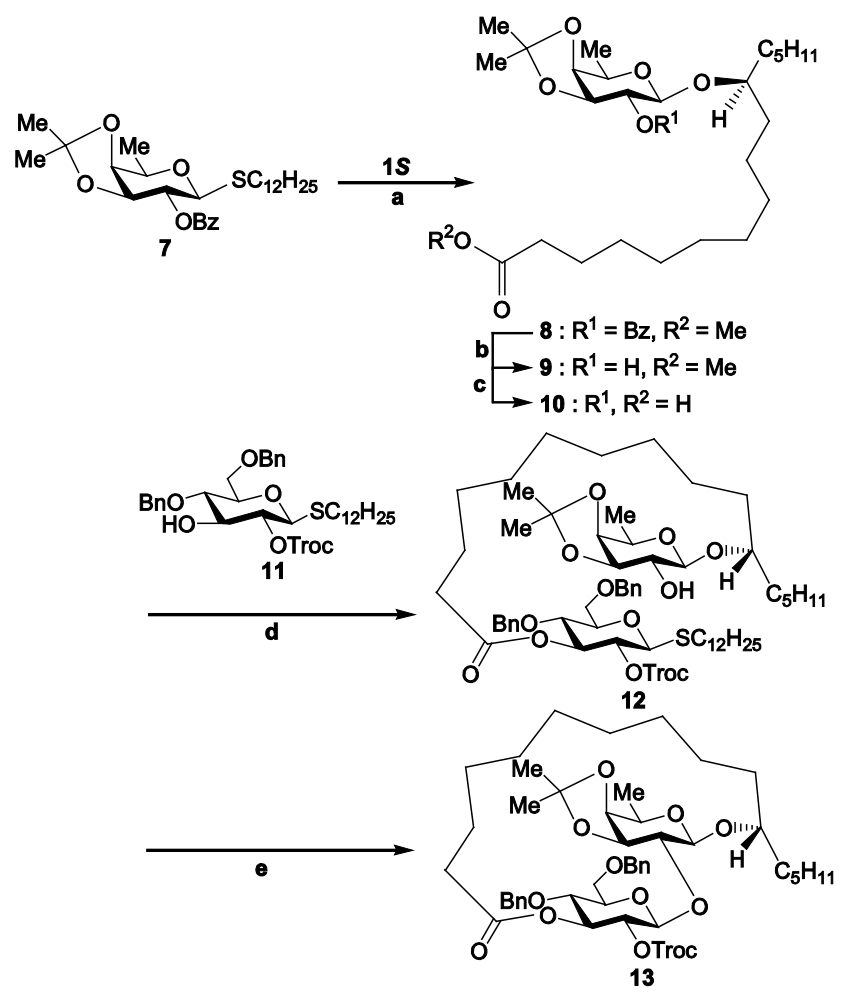

Scheme 2 Synthesis of a disaccharide constituent of tricolorin A 13. Reagents and conditions: (a) MeOTf, MS $4 \AA$ A, $\mathrm{CH}_{2} \mathrm{Cl}_{2}$, r.t., 1 h, 81\%; (b) $\mathrm{NaOMe}, \mathrm{MeOH}$, 


\section{Scheme 3}
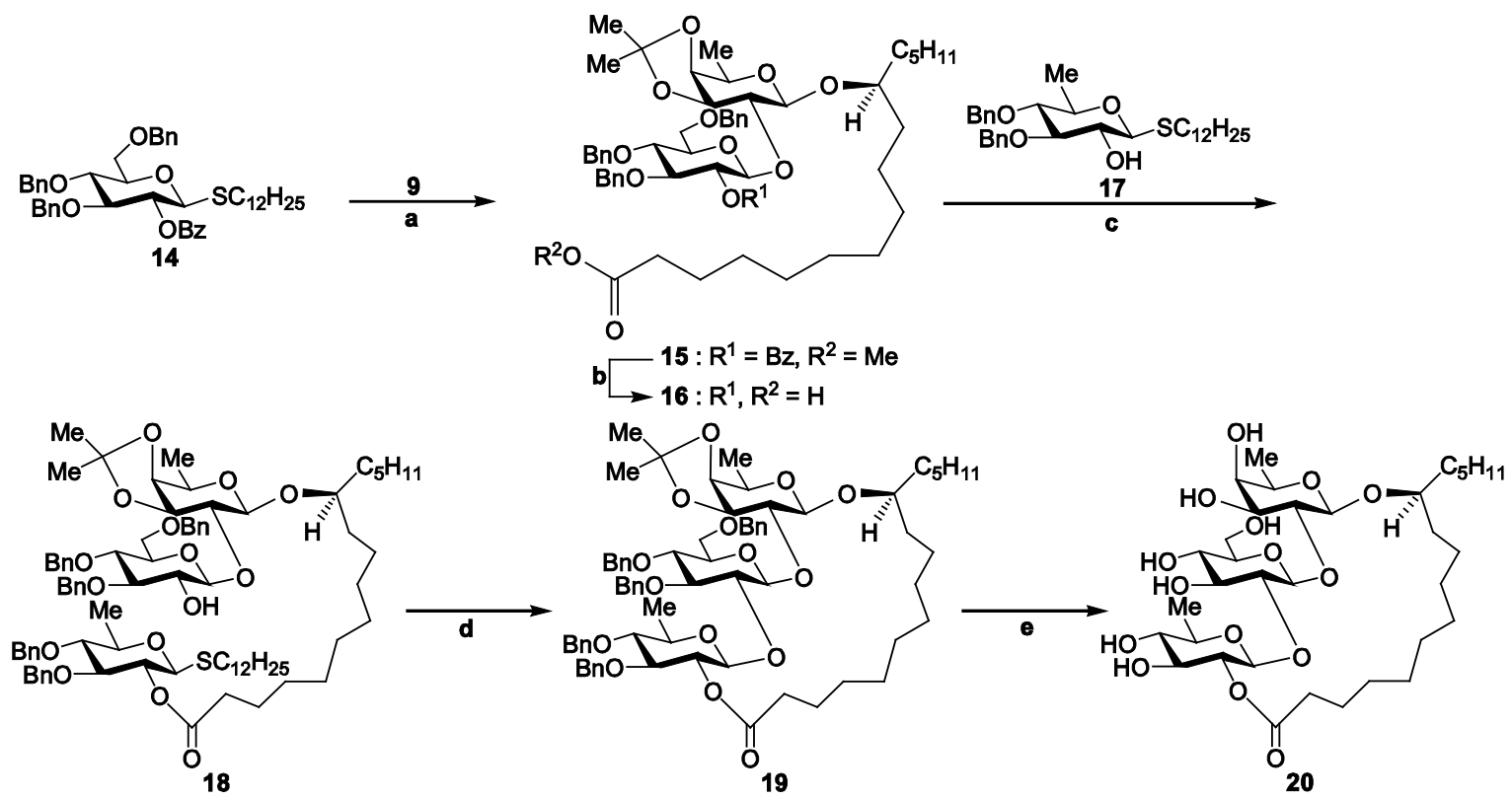

Scheme 3 Total synthesis of tricolorin F. Reagents and conditions: (a) $\mathrm{MeOTf}, \mathrm{MS} 4 \AA \mathrm{A}^{-} \mathrm{CH}_{2} \mathrm{Cl}_{2}$, r.t., 6 h, 83\%; (b) $\mathrm{NaOH}, \mathrm{THF}-\mathrm{EtOH}, 50{ }^{\circ} \mathrm{C}, 4$ h, 99\%; (c) WSC $\cdot \mathrm{HCl}, \mathrm{DMAP}, \mathrm{CH}_{2} \mathrm{Cl}_{2}$, r.t., 24 h, $72 \%$; (d) MeOTf, $\mathrm{MS} 4 \AA$, $\mathrm{CH}_{2} \mathrm{Cl}_{2}$, r.t., 24 h, 70\%; (e) i) $\mathrm{HCl}, \mathrm{MeOH}$, r.t., 2 h; ii) $\mathrm{Pd} / \mathrm{C}, \mathrm{H}_{2}, \mathrm{MeOH}$, r.t., 24 h, $99 \%$. 


\section{Table 1}

Results of intramolecular glycosylation ${ }^{a}$

\begin{tabular}{llllll}
\hline Intry & Promoter & \multicolumn{1}{l}{$\begin{array}{l}\text { Concentration } \\
\text { of } 5\end{array}$} & Time & Yield & $R / \mathcal{S}^{\mathrm{c}}$ \\
\hline 1 & MeOTf(10 equiv) & $2.2 \mathrm{mM}$ & $19 \mathrm{~h}$ & $69 \%$ & $52 / 48$ \\
2 & MeOTf (5 equiv) & $4.5 \mathrm{mM}$ & $13 \mathrm{~h}$ & $63 \%$ & $45 / 55$ \\
3 & MeOTf (5 equiv) & $8.9 \mathrm{mM}$ & $13 \mathrm{~h}$ & $39 \%$ & $29 / 71$ \\
4 & MeOTf (5 equiv) & $17.8 \mathrm{mM}$ & $13 \mathrm{~h}$ & $35 \%$ & $22 / 78$ \\
5 & NIS (1.5 equiv) & $4.5 \mathrm{mM}$ & $13 \mathrm{~h}$ & $52 \%$ & $41 / 59$ \\
\hline a Reactions were performed in dry $\mathrm{CH}_{2} \mathrm{Cl}_{2}$ at r.t. for MeOTf and at $-20^{\circ} \mathrm{C}$ for NIS. \\
b Catalytic amount of TfOH was added together with NIS. \\
\multicolumn{7}{c}{ ' Ratio of diastereomer was determined by ${ }^{1} \mathrm{H}$ NMR analysis. }
\end{tabular}

